\section{Response to Ballabh and LaGamma}

Advance online publication 24 September 2014. doi:10.1038/pr.2014.112

To the Editor: We would like to thank Drs Ballabh and LaGamma for their comments (1) regarding our article (2) on the effects of glycerol on the developing brain. We agree that the glycerol-induced intraventricular hemorrhage model has shown the importance of blood on hindering the development of oligodendrocytes and being a cause of white matter injury. Their complementary work using cerebral slices in the absence of glycerol has yielded similar results as the in vivo model thereby strengthening the findings of the glycerol injection model. However, we could not rule out the possibility that glycerol was decreasing proliferation in the cerebellum.

Glycerol has known anti-proliferative effects on many cell types $(3,4)$. Neurogenesis within the cerebrum is for the most part completed by the time frame of interest. In contrast, the cerebellum is undergoing massive neurogenesis during the time of glycerol exposure. The decrease in cerebellar size as the dose of glycerol was increased, without differences in rates of intraventricular hemorrhage or body growth between glycerol-exposed kits makes glycerol suspect. The increase in glycerol concentration in the cerebellum further supports glycerol as a culprit.

We saw very few early deaths at less than $48 \mathrm{~h}$, as most of the deaths occurred between days 3 and 5 . We did not see any seizures at all in our study and this is likely due to our low rate of intraventricular hemorrhage, confirmed by ultrasound and at necropsy. Animals that were found dead had bottled fed perfectly the feeding before death. No evidence of milk in the lungs was ever found. However, the intestines showed evidence of pneumatosis. We were unable to tell time of death so these findings may be normal post mortem changes and not necrotizing enterocolitis. Nipples were cleaned between pups, but the fact that they were bottled fed instead of gavage fed may have been a contributing factor.
We are not the only group to see that intraventricular hemorrhage is not consistent in rabbits. Coulter et al. had no intraventricular hemorrhage after 225 kits with all of them receiving multiple doses of glycerol and additional agents (5). The fact that we obtained our animals from a different vendor may be the biggest difference in the study as Coulter et al. were based in Utah while Lorenzo et al. were in Boston $(5,6)$.

After developing a new mouse model of posterior fossa hemorrhage, we believe that subarachnoid hemorrhages may account for or contribute to the cerebellar changes we noted using the glycerol model, as we are seeing similar cerebellar pathology after exposure to only blood. While we acknowledge the importance of cerebral findings of both pathology and neuroprotection by Dr Ballabh' group using the glycerol-induced IVH model, we have found that direct injection of blood into the posterior fossa provides a more consistent model for studying cerebellar injury.

Disclosure: There is no conflict of interest to disclose.

\section{Christopher M. Traudt ${ }^{1}$ and Sandra E. Juul ${ }^{1}$}

${ }^{1}$ Department of Pediatrics, University of Washington, Seattle, Washington.

Correspondence: Christopher M. Traudt (ctraudt@uw.edu)

\section{REFERENCES}

1. Ballabh P, LaGamma EF. Strategies for working with a preterm rabbit model of glycerol-induced intraventricular hemorrhage: strengths and limitations Pediatr Res 2014; (this issue).

2. Traudt CM, McPherson RJ, Studholme C, Millen KJ, Juul SE. Systemic glycerol decreases neonatal rabbit brain and cerebellar growth independent of intraventricular hemorrhage. Pediatr Res 2014;75:389-94.

3. Wiebe JP, Dinsdale CJ. Inhibition of cell proliferation by glycerol. Life Sci 1991;48:1511-7.

4. Sugiyama N, Mizuguchi T, Aoki T, et al. Glycerol suppresses proliferation of rat hepatocytes and human HepG2 cells. J Surg Res 2002;103:236-42.

5. Coulter DM, LaPine T, Gooch WM 3rd. Intraventricular hemorrhage in the premature rabbit pup. Limitations of this animal model. J Neurosurg 1984;60:1243-5.

6. Lorenzo AV, Welch $\mathrm{K}$, Conner S. Spontaneous germinal matrix and intraventricular hemorrhage in prematurely born rabbits. J Neurosurg 1982;56:404-10. 